\title{
Nanostructured Aerosol Particles: Fabrication, Pulmonary Drug Delivery, and Controlled Release
}

\author{
Xingmao Jiang, ${ }^{1,2}$ Yung-Sung Cheng, ${ }^{2}$ and Hugh D. C. Smyth ${ }^{3}$ \\ ${ }^{1}$ Department of Chemical and Nuclear Engineering and Center for Micro-Engineered Materials, \\ The University of New Mexico, Albuquerque, NM 87106, USA \\ ${ }^{2}$ Aerosol and Respiratory Dosimetry Program, Lovelace Respiratory Research Institute, Albuquerque, NM 87108, USA \\ ${ }^{3}$ Division of Pharmaceutics, College of Pharmacy, The University of Texas at Austin, Austin, TX 78712, USA \\ Correspondence should be addressed to Xingmao Jiang, xjiang@lrri.org
}

Received 3 March 2011; Accepted 3 March 2011

Copyright (c) 2011 Xingmao Jiang et al. This is an open access article distributed under the Creative Commons Attribution License, which permits unrestricted use, distribution, and reproduction in any medium, provided the original work is properly cited.

Pulmonary drug delivery is the preferred route of administration in the treatment of respiratory diseases and some nonrespiratory diseases. Recent research has focused on developing structurally stable high-dosage drug delivery systems without premature release. To maximize the deposition in the desired lung regions, several factors must be considered in the formulation. The special issue includes seven papers deal with aerosol-assisted fabrication of nanostructured particles, aerosol deposition, nanoparticles pulmonary exposure, and controlled release.

The delivery by inhalation utilizes the extensive surface area of the alveoli, avoiding hepatic first-pass metabolism and enabling noninvasive administration of larger doses to the lungs, leading to greater therapeutic efficacy without increasing toxicity. Pulmonary drug delivery is the preferred route of administration of aerosolized drugs in the treatment of respiratory diseases including asthma and cystic fibrosis, infectious diseases, in particular tuberculosis, and some nonrespiratory diseases such as type I diabetes. Recent research has focused on developing structurally stable highdosage drug delivery systems without any premature release. To maximize the deposition in desired lung regions, a formulation must be carefully engineered to ensure the carriers have appropriate aerodynamic diameter, size distribution, porosity, density, surface chemistry for optimal dispersibility, and lung targeting. To overcome the problems with the instability of the formulation and poor loading efficiencies, sol gel approaches and wet chemistry have been applied for fabrication of nanocomposite particles with corrugated surfaces, reduced surface energy, and adjustable hydrophobicity/hydrophilicity to improve particle dispersion.

The seven papers presented in this special issue investigate aerosol-assisted fabrication of nanostructured particles, aerosol deposition, nanoparticle pulmonary exposure, and controlled release.
In the first paper, cerium corrosion inhibitors are encapsulated into hexagonally ordered nanoporous silica particles via single-step aerosol-assisted self-assembly. The core/shell structured particles are effective for corrosion inhibition of aluminum alloy AA2024-T3. The release can be controlled by the pore size, pore surface chemistry, and the inhibitor solubility.

In the second paper, azobenzene ligands are uniformly anchored to the pore surfaces of nanoporous silica particles with single-crystal $\mathrm{NaCl}$. The addition of amphiphilic solvents into the release medium delays $\mathrm{NaCl}$ release. The functionalized particles demonstrate a photocontrolled release by trans/cis isomerization of azobenzene moieties.

In the third paper, hydrogel particles are produced from sprayed polymeric nano/microdroplets by a nebulization process that is immediately followed by gelation in a crosslinking fluid. Particle synthesis parameters such as type of nebulizer, type of crosslinker, air pressure, and polymer concentration are investigated for their impact on the mean particle size, swelling behavior, and morphology of the developed particles.

In the fourth paper, smart magnetically responsive hydrogel nanoparticles are prepared by a novel spray gelation-based method for biomedical and drug delivery applications. Oligoguluronate and PEG-grafted chitosan 
are synthesized and characterized. Magnetically responsive hydrogel nanoparticles based on alginate and alginate/Gblocks are synthesized via aerosolization followed by either ionotropic gelation or both ionotropic and polyelectrolyte complexation using $\mathrm{CaCl}_{2}$ or PEG-g-chitosan/ $\mathrm{CaCl}_{2}$ as crosslinking agents.

In the fifth paper, the authors study the feasibility of magnetically targeted delivery of high aspect ratio particles loaded with iron-oxide nanoparticles to the site of the tumour. Iron sensitive magnetic resonance images of the lungs are acquired to determine the iron concentrations in the right and left lung of each animal. The right/left ratio increases in the middle and basal regions of the lung. It is anticipated that with further optimization, this technique could be an effective method for increasing the dose of drug delivered to a specific site within the lung.

In the sixth paper, nanoparticles instilled through both an intratracheal tube and an inhalation system can exacerbate the lung inflammation by bacterial endotoxin. Repetitive pulmonary exposure to nanoparticles has aggravating effects on allergic airway inflammation, including adjuvant effects on Th2-milieu. Nanoparticle exposure may synergistically facilitate pathological inflammatory conditions in the lung via both innate and adaptive immunological abnormalities.

In the final paper, controlled release of inhaled nanoparticles is evaluated using isolated, perfused, and ventilated lung models. Ex vivo models are thought to overcome the common obstacles of in vitro tests and offer more reliable drug release and distribution data that are closer to the in vivo situation. 

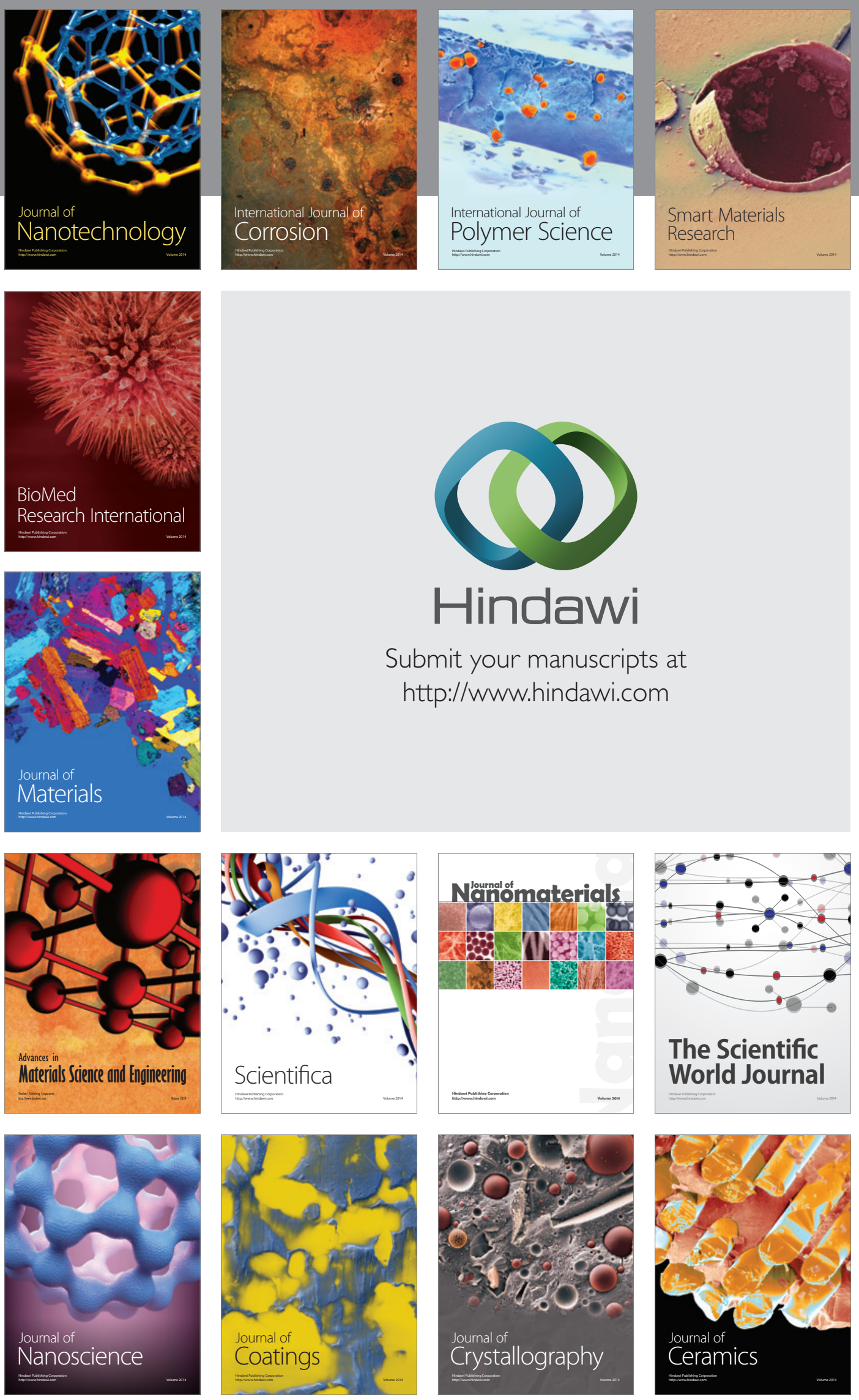

The Scientific World Journal

Submit your manuscripts at

http://www.hindawi.com

\section{World Journal}

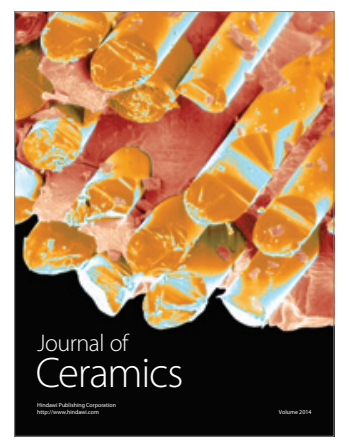

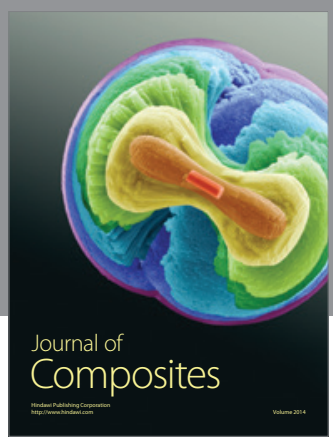
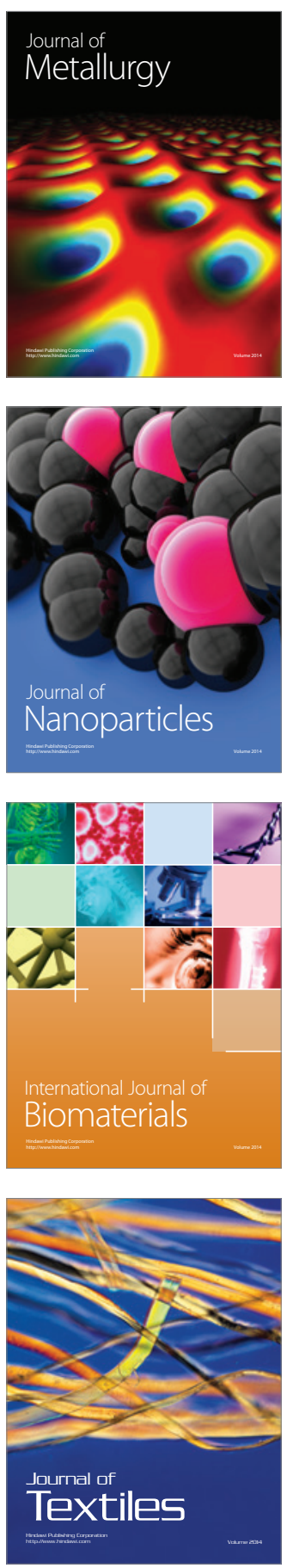